\title{
Planning and Design of Robotic Production
}

\author{
Luis Fernando Roque Vidal ${ }^{1}$, and $\operatorname{Olg} a$ Ogorodnikova ${ }^{1, *}$ \\ ${ }^{1}$ Ural Federal University, 620002 Yekaterinburg, Russia
}

\begin{abstract}
This paper discusses the design of automated lines, taking into account the use of industrial robots to perform technological operations. When planning manufacturing processes, as well as the placement of process equipment and robots in the production area, optimization techniques are usually used to ensure critical performance and efficiency indicators. The considered design methods are shown on the example of an automated line for the production of lithium-ion batteries. To design this line, 3D CAD models of technological equipment and transport lines were built. Based on the results of time analysis, those technological operations were opted, for which it would be advisable to use robots. The design of the robotic production for the technological operations was carried out using $\mathrm{ABB}$ robots. The robots required were chosen from the $\mathrm{ABB}$ catalog by weight, size of work area, speed and power. The plan of the robotic zones was drawn up using the ABB RobotStudio software, which allows visualizing the movements and programming robots off-line.
\end{abstract}

\section{Introduction}

Each year, the demand for lithium-ion batteries increases as new portable electronic devices continue to appear in the market [1]. Lithium-ion batteries are used not only for smart phones and laptops, but also in mobile robots, electric vehicles, data centre servers, and many other industrial applications. The demand for autonomous power supplies based on the lithium-ion batteries is expected to grow [2], which determines the relevance of this work regarding the manufacture of the batteries.

When planning production, automated lines for the manufacture of batteries using robots are of interest. At the early stage of designing an automated line, the issue of choosing technological operations is important, where human labor must be replaced by a robot [3]. Robots are commonly used to perform high-precision or monotonous repetitive operations, in addition, robots increase productivity, reduce scrap and lower product costs. At the same time, since modern production systems are difficult to analyze due to their complex structure, methods of mathematical modeling and computer simulation can help to study them. There are special software tools to simulate production processes, such as ARENA, FlexSim, SIMIO and others. Among others, the ABB RobotStudio [4] from the manufacturer of a wide range of industrial robots should be highlighted for the ability to directly select the required robots and apply them on the automated line at the stage of conceptual planning and simulation [5].

*Corresponding author: Olga.Ogorodnikova@urfu.ru 


\section{Planning of the automated line}

Planning of the automated line assumes initial analysis of technological operations, including execution time as well as the possibility to automate the process and use robots. The automated line includes technological equipment, transport systems and storage areas. Table 1 shows the listing of technological operations for manufacturing lithium-ion batteries and useful information for the analysis.

Table 1. Manufacturing diagram of the lithium-ion batteries production.

\begin{tabular}{|c|c|c|c|c|c|c|c|c|}
\hline $\mathrm{N}$ & Manufacturing Process* & $\mathrm{O}$ & $\mathrm{D}$ & $\mathrm{T}$ & $\mathrm{S}$ & $\mathrm{R}$ & $\begin{array}{l}\text { Time } \\
(\min )\end{array}$ & $\begin{array}{l}\text { Distance } \\
(\mathrm{m})\end{array}$ \\
\hline 1 & Transportation of the raw material & & & & & $\mathrm{M}$ & 5 & 5 \\
\hline 2 & Mixing of the lithium carbonate & & & & & $\mathrm{A}$ & 30 & \\
\hline 3 & Charging the slurry to the coating machine & & & & & $\mathrm{M}$ & 1 & 5 \\
\hline 4 & Coating of the slurry into the copper rolls & & & & & $\mathrm{A}$ & 6 & \\
\hline 5 & Drying of the rolls & & & & & $\mathrm{A}$ & 5 & \\
\hline 6 & Taking the rolls to the calendering machine & & & & & $\mathrm{M}$ & 5 & 2 \\
\hline 7 & Thickening of the rolls in the calendering machine & & & & & $\mathrm{A}$ & 7 & \\
\hline 8 & Taking the rolls into the slitting machine & & & & & $\mathrm{M}$ & 2 & 2 \\
\hline 9 & Cutting the rolls into smaller rolls & & & & & $\mathrm{A}$ & 7 & \\
\hline 10 & Charging the rolls into the vacuum oven & & & $>$ & & $\mathrm{M}$ & 1 & 2 \\
\hline 11 & Vacuum drying of the rolls & & & & & $\mathrm{A}$ & 120 & \\
\hline 12 & Taking the rolls into the separation machine & & & & & $\mathrm{M}$ & 1 & 2 \\
\hline 13 & Cutting the rolls into sheets for the cells & & & & & $\mathrm{A}$ & 22 & \\
\hline 14 & Charging the stacking machine with the sheets & & & & & $\mathrm{M}$ & 3 & \\
\hline 15 & Stacking of the sheets with the separator & & & & & $\mathrm{A}$ & 1 & \\
\hline 16 & Transportation of the stacked sheets to the & & & & & $\mathrm{R}$ & 0.25 & 1 \\
\hline 17 & Moving the stacked sheets on the conveyor & & & & & $\mathrm{A}$ & 0.5 & 3 \\
\hline 18 & Wielding the sheets with the connectors & & & & & $\mathrm{M}$ & 1 & \\
\hline 19 & Moving the stacked sheets on the conveyor & & & & & $\mathrm{A}$ & 0.5 & 3 \\
\hline 20 & Sealing the sheets with the case of the battery & & & & & $\mathrm{M}$ & 1 & \\
\hline 21 & Moving the battery on the conveyor & & & & & $\mathrm{A}$ & 0.5 & 3 \\
\hline 22 & Filling the battery with the electrolyte & & & & & $\mathrm{M}$ & 2 & \\
\hline 23 & Moving the battery on the conveyor & & & & & $\mathrm{A}$ & 0.32 & 6 \\
\hline 24 & Packing the batteries into boxes & & & & & $\mathrm{R}$ & 0.16 & \\
\hline 25 & Loading the batteries into the forming machines & & & & & $\mathrm{M}$ & 5 & \\
\hline 26 & Forming of the batteries & & & & & $\mathrm{A}$ & 1440 & \\
\hline
\end{tabular}

* Simbols in the Table 1 mean as follows: O is Operation, T is Transportation, D is Delay, M is Manual operation, A is Automated operation, and R is Robotic operation.

Execution time and distances for transportation, seen in the manufacturing diagram, are estimated based on the investigation of technological operations and equipment characteristics. Preliminary plan of the plant was drawnn up based on the information about the number and size of the equipment. According to the diagram, the total time to make one battery is approximately $28 \mathrm{hrs}$, and it needs 10 transportations for 13 operations, 1 storage 
for one delay. Analyzing the manufacturing diagram, it is possible to highlight those technological operations that can be automated using small or collaborative robots

Figure 1 shows the 3D scheme of the plant for production of lithium-ion batteries, created in $\mathrm{ABB}$ RobotStudio, and also in more detail the area with $3 \mathrm{D}$ models of equipment, which were created using $\mathrm{CAD}$ AutoCAD and loaded to $\mathrm{ABB}$ Robotstudio. The $3 \mathrm{D}$ models of the required small robots were chosen from the $\mathrm{ABB}$ data base and placed into the scheme as well. Section 1 on the scheme is intended for manufacturing anodes and cathodes of the lithium-ion battery cells, section 2 of the plant is for assembling the batteries, section 3 is for charging the assembled batteries.

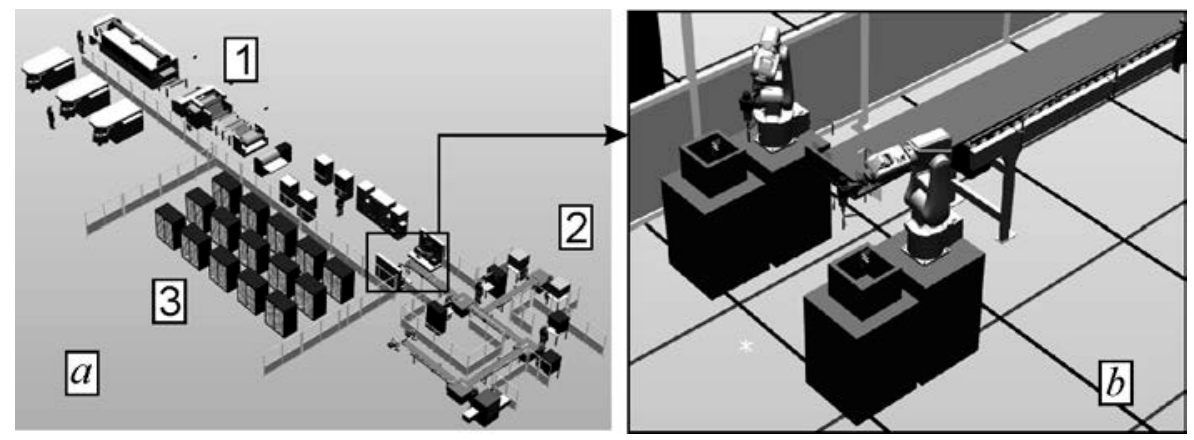

Fig. 1. 3D scheme of the plant for production of lithium-ion batteries created in ABB RobotStudio (a) and $\mathrm{ABB}$ robots applied on the automated line (b).

In the section 2 , the robots are placed next to the stacking machine, from where they pick up the battery cells as soon as the operation of sheet stacking operation is completed. Unfortunately, RobotStudio doesn't load all the details of the 3D models created in AutoCAD for the stacking machines, nevertheless, the actions become more noticeable after starting the simulation.

\section{Installation of robots on the automated line}

When planning and designing an automatic line, an assembly operation was opted to install robots due to the repeatability and simplicity of the tasks performed. Since the assembly starts with the stacking process, it is necessary to automate this section as much as possible, in particular, to install conveyors for transportation of batteries from the stacking machines and between assembly operations. We estimate that four robots can synchronously perform assembly operations on such conveyor line, given that semi-automatic machines and human operators also do the work on the line. Location of four robots and five conveyors in the designed 3D scheme is shown in fig. 2.

The tasks assigned to robots are as follows. 1) To take a cell with the sheets of anodes and cathodes already stacked and packed and place it on the conveyor (stacking area). It is simply moving parts from one place to another, too much repetitive and monotonously tedious for a human operator. 2) To take the finished battery that comes to the conveyor place and put it into a box (assembly area). To ensure transportation of batteries on the production line, they must be gathered into boxes.

The tasks assigned to the conveyors are as follows. 1) To transport the cell after stacking operation to the location of wielding machines, then the robot places the cell on the conveyor and the operator picks it up. 2) To transport the aluminium case from the case forming location to the assembling. 3) To transport the cell from the wielding location, receive the aluminium case and transport all the parts together to the sealing location, where 
an operator have to take both, the cell and the case, from the conveyor to perform next operation. 4) To transport the battery from the sealing location to the electrolyte filling location. 5) To transport the battery from the filling location to the packing location where the operator puts the battery on the conveyor, and at the end of it the robot takes the battery and place it into the box.

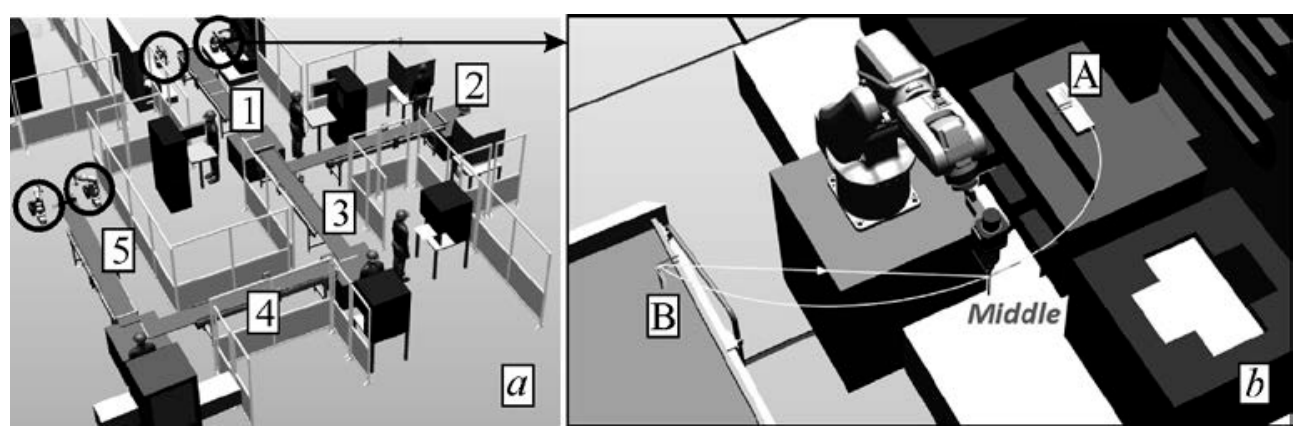

Fig. 2. Location of robots and conveyors along the automated line (a) and targets of the robot (b).

For the designed line, the ABB IRB-120 robots were opted. This type of robots is one of the latest additions to the ABB family. The robot can withstand a load up to $3 \mathrm{~kg}$, since the batteries don't weight more than $0.3 \mathrm{~kg}$, the task of moving them is an achievable task. The robot is specifically designed for small to medium industries that require flexible automation. It has 6 axes of movement that enable it to have enough freedom of moving cells and batteries so that to place them on the conveyor or into the box.

\section{Conclusion}

The plant with automated line is designed for production of lithium-ion batteries using robots to perform selective technological operation. ABB robots and ABB RobotStudio software tools are used to plan the manufacturing process. The production line proposed in this project is a completely new alternative for the manufacturing of lithium-ion batteries. While not as advanced or large compared to other mega-factories around the world, it is cheaper thanks to the use of semi-automatic machines in collaboration with human operators and robots for maximum efficiency.

\section{References}

1. G. Zubi, R. Dufo-López, M. Carvalho, G. Pasaoglu, Renewable and Sustainable Energy Reviews 89, 292-308 (2018)

2. D. Coffin, J. Horowitz, Journal of International Commerce and Economics 332, 1-21 (2018)

3. P. Barosz, G. Gołda, A. Kampa, Applied Sciences 10, 2862 (2020)

4. L. F. Roque Vidal, O. M. Ogorodnikova, AIP Conference Proceedings 2313, 040008 (2020)

5. O. M. Ogorodnikova, K. A.Vaganov, I. D. Putimtsev, AIP Conference Proceedings 1886, 020017 (2017) 